University of Nebraska - Lincoln

DigitalCommons@University of Nebraska - Lincoln

May 1975

\title{
Effects of anisotropic electron-ion interactions in atomic photoelectron angular distributions
}

\author{
Dan Dill \\ Boston University, Boston, Massachusetts \\ Anthony F. Starace \\ University of Nebraska-Lincoln, astarace1@unl.edu \\ Steven T. Manson \\ Georgia State University, Atlanta, Georgia
}

Follow this and additional works at: https://digitalcommons.unl.edu/physicsstarace

Part of the Physics Commons

Dill, Dan ; Starace, Anthony F.; and Manson, Steven T., "Effects of anisotropic electron-ion interactions in atomic photoelectron angular distributions" (1975). Anthony F. Starace Publications. 5.

https://digitalcommons.unl.edu/physicsstarace/5

This Article is brought to you for free and open access by the Research Papers in Physics and Astronomy at DigitalCommons@University of Nebraska - Lincoln. It has been accepted for inclusion in Anthony F. Starace Publications by an authorized administrator of DigitalCommons@University of Nebraska - Lincoln. 


\title{
Effects of anisotropic electron-ion interactions in atomic photoelectron angular distributions
}

\author{
Dan Dill \\ Department of Chemistry, Boston University, Boston, Massachusetts 02215 \\ Anthony F. Starace* \\ Behlen Laboratory of Physics, The University of Nebraska, Lincoln, Nebraska 68508
}

Steven T. Manson ${ }^{\dagger}$

Department of Physics, Georgia State University, Atlanta, Georgia 30303

(Received 3 October 1974)

\begin{abstract}
The photoelectron asymmetry parameter $\beta$ in $L S$ coupling is obtained as an expansion into contributions from alternative angular-momentum transfers $j_{t}$. The physical significance of this expansion of $\beta$ is shown to be that (i) the electric-dipole interaction transfers to the atom a characteristic single angular momentum $j_{t}=l_{0}$, where $l_{0}$ is the photoelectron's initial orbital momentum, whereas (ii) angular-momentum transfers $j_{t} \neq l_{0}$ indicate the presence of anisotropic (i.e., term-dependent) interaction of the outgoing photoelectron with the residual ion. For open-shell atoms the photoelectron-ion interaction is generally anisotropic; photoelectron phase shifts and electric-dipole matrix elements depend on both the multiplet term of the residual ion and the total orbital momentum of the ion-photoelectron final-state channel. Consequently, $\beta$ depends on the term levels of the residual ion and contains contributions from all allowed values of $j_{t}$. These findings contradict the independent-particle-model theory for $\beta$, which ignores the final-state electron-ion interaction and to which our expressions reduce in the limiting cases for which only $j_{t}=l_{0}$ is allowed, namely (a) spherically symmetric atoms (e.g., closed-shell atoms) and (b) open-shell atoms for which the electron-ion interaction is isotropic (e.g., very light elements). Numerical calculations of the asymmetry parameters and partial cross sections for photoionization of atomic sulfur are presented to illustrate the theory and to demonstrate the information on electron-ion dynamics that can be obtained from the theoretical and experimental study of $\beta$ for open-shell atoms.
\end{abstract}

\section{INTRODUCTION}

We obtain in this paper explicit expressions, in $L S$ coupling, for the angular distribution of photoelectrons produced by electric dipole ionization of an arbitrary open- or closed-shell atom. Our treatment is based on the angular momentum transfer expansion for the differential photoionization cross section ${ }^{1-3}$ and is intended to provide a theoretical framework that allows angular distribution calculations comparable in accuracy to the best calculations of total photoionization cross sections. The formulas we obtain show explicitly the influence of anisotropic electron-ion interactions on the electron angular distribution and at the same time explain the success of the Cooper$\mathrm{Zare}^{4}$ independent-particle-model theory in predicting such distributions for closed-shell atoms. ${ }^{5,6}$ For other than the lightest open-shell atoms, ${ }^{7}$ however, we expect anisotropic electron-ion interactions to produce photoelectron angular distributions that deviate significantly from the predictions of the Cooper-Zare theory.

Our conclusions, described above, are contained implicitly in the $L S$-coupling formulas for the angular distribution asymmetry parameter $\beta$ obtained by Lipsky ${ }^{8,9}$ and by Jacobs and Burke, ${ }^{10}$ whose formulas are in principle equivalent to ours. The advantage of the angular momentum transfer expansion employed in this paper, however, is that such conclusions follow explicitly from our formulation. Hartree-Fock calculations of the angular distribution of electrons photoionized from atomic sulfur, a typical open-shell atom, are presented to illustrate our theoretical predictions.

In Sec. II we summarize the angular momentum transfer formulation of the differential photoionization cross section. We also exhibit how the angular momentum transfer probes anisotropic electron-ion interactions. The formulas in this section depend on the amplitude for photoionization with a particular value of the angular momentum transfer. The form of this amplitude in LS coupling, a main result of this paper, is obtained in Sec. III. In Sec. IV we illustrate the theory by calculating the photoelectron angular distribution of atomic sulfur. Lastly, we discuss our conclusions in Sec. V. A brief report of these results has been published elsewhere. ${ }^{11}$ 


\section{SUMMARY OF THE ANGULAR MOMENTUM TRANSFER FORMULATION}

The ejection of an electron $e^{-}$from an unpolarized atomic target $A$ by electric dipole interaction with an incident photon $\gamma$ may be represented schematically as

$$
\begin{aligned}
A\left(J_{0} \pi_{0}\right)+\gamma\left(j_{\gamma}=1,\right. & \left.\pi_{\gamma}=-1\right) \\
& -A^{+}\left(J_{c} \pi_{c}\right)+e^{-\left[l s j, \pi_{e}=(-1)^{l}\right] .}
\end{aligned}
$$

The differential cross section for this process can be separated into contributions characterized by alternative values of the angular momentum transfer,

$$
\overrightarrow{\mathrm{j}}_{t}=\overrightarrow{\mathrm{j}}_{\gamma}-\overrightarrow{\mathrm{l}}=\overrightarrow{\mathrm{J}}_{c}+\overrightarrow{\mathrm{s}}-\overrightarrow{\mathrm{J}}_{0},
$$

provided no measurement is made of either the photoelectron spin or the orientation of the residual ion. The vector $\vec{j}_{t}$ is the angular momentum transferred between the unobserved initial and final angular momenta in the reaction, i.e., between the total angular momentum $\vec{J}_{0}$ of the target $A$ and the combined angular momenta of the residual ion $A^{+}$and the photoelectron spin $\overrightarrow{\mathbf{s}}$, which we denote $\vec{J}_{c s} \equiv \vec{J}_{c}+\vec{s}$. Allowed values of $j_{t}$ are determined by conservation of angular momentum $\vec{J}$ and parity $\pi$ in reaction (1):

$$
\begin{aligned}
& \overrightarrow{\mathrm{J}}=\overrightarrow{\mathrm{J}}_{0}+\overrightarrow{\mathrm{j}}_{\gamma}=\overrightarrow{\mathrm{J}}_{c}+\overrightarrow{\mathrm{s}}+\overrightarrow{\mathrm{l}}, \\
& \pi=-\pi_{0}=\pi_{c}(-1)^{l} .
\end{aligned}
$$

The general form of the differential cross section for reaction (1) is ${ }^{12}$

$$
\frac{d \sigma}{d \Omega}=\frac{\sigma}{4 \pi}\left[1+\beta P_{2}(\cos \theta)\right] .
$$

Here $\sigma$ is the total cross section, $\theta$ is the angle between the axis of linear polarization of the incident light and the direction of the outgoing photoelectron, and $\beta$ is the asymmetry parameter. The dynamical features of the angular distribution are thus contained in $\beta$, which may assume values in the range $-1 \leqslant \beta \leqslant 2$, corresponding to distributions varying from $\sin ^{2} \theta$ to $\cos ^{2} \theta$. [Though $\mathrm{Eq}$. (5) assumes linearly polarized incident light, unpolarized, ${ }^{13}$ partially polarized, ${ }^{14}$ and elliptically polarized $^{15}$ incident light produce angular distributions that may be expressed in terms of $\beta$.]

The resolution of Eq. (5) into contributions corresponding to alternative values of $j_{t}$ requires first that one determine the allowed values of $j_{t}$ from Eqs. (2)-(4). Second, each value of $j_{t}$ is characterized as being either parity favored or parity unfavored, ${ }^{2}$ corresponding to whether the parity change of the target $\pi_{0} \pi_{c}$ is equal to $+(-1)^{j} t$ or $-(-1)^{j} t$, respectively. The total cross section $\sigma$ and the asymmetry parameter $\beta$ may then be ex- pressed in terms of cross sections $\sigma\left(j_{t}\right)$ and asymmetry parameters $\beta\left(j_{t}\right)$ for a particular value of $j_{t}$ as follows ${ }^{3}$ :

$$
\begin{aligned}
& \sigma=\sum_{j_{t}, J_{c s}} \sigma\left(j_{t}\right), \\
& \sigma \beta=\sum_{J_{c s}}\left(\sum_{j_{t}}^{\text {fav }} \sigma\left(j_{t}\right)_{\mathrm{fav}} \beta\left(j_{t}\right)_{\mathrm{fav}}-\sum_{j_{t}}^{\text {unf }} \sigma\left(j_{t}\right)_{\mathrm{unf}}\right) .
\end{aligned}
$$

In Eq. (7) we have summed the favored and unfavored values of $j_{t}$ separately, but, as seen from Eq. (11) below, Eq. (7) represents $\beta$ as a weighted average of $\beta\left(j_{t}\right)$. Note that while Eqs. (6) and (7) also have sums over $J_{c s}$ (cf. Ref. 3, p. 1981) we do not indicate the dependence of $\sigma\left(j_{t}\right)$ and $\beta\left(j_{t}\right)$ on quantum numbers other than $j_{t}$ until Sec. III of this paper. This dependence is hidden in the scattering matrix amplitudes $\bar{S}_{l}\left(j_{t}\right)$, in terms of which $\sigma\left(j_{t}\right)$ and $\beta\left(j_{t}\right)$ are given by ${ }^{3}$

$$
\begin{aligned}
\sigma\left(j_{t}\right)_{\mathrm{fav}}= & \pi \chi^{2} \frac{2 j_{t}+1}{2 J_{0}+1}\left[\left|\bar{S}_{+}\left(j_{t}\right)\right|^{2}+\left|\bar{S}_{-}\left(j_{t}\right)\right|^{2}\right] \\
\sigma\left(j_{t}\right)_{\mathrm{unf}}= & \pi \chi^{2} \frac{2 j_{t}+1}{2 J_{0}+1}\left|\bar{S}_{0}\left(j_{t}\right)\right|^{2} \\
\beta\left(j_{t}\right)_{\mathrm{fav}}= & \left\{\left(j_{t}+2\right)\left|\bar{S}_{+}\left(j_{t}\right)\right|^{2}+\left(j_{t}-1\right)\left|\bar{S}_{-}\left(j_{t}\right)\right|^{2}\right. \\
& \left.-3\left[j_{t}\left(j_{t}+1\right)\right]^{1 / 2}\left[\bar{S}_{+}\left(j_{t}\right) \bar{S}_{-}\left(j_{t}\right)^{+}+\text {c.c. }\right]\right\} \\
& \left\{\left(2 j_{t}+1\right)\left[\left|\bar{S}_{+}\left(j_{t}\right)\right|^{2}+\left|\bar{S}_{-}\left(j_{t}\right)\right|^{2}\right]\right\} \\
\beta\left(j_{t}\right)_{\mathrm{unf}}= & -1
\end{aligned}
$$

In the se equations, $x$ is the photon wavelength divided by $2 \pi$ and c.c. denotes complex conjugate. The parity-favored cross sections and asymmetry parameters, Eqs. (8) and (10), depend on photoionization amplitudes $\bar{S}_{ \pm}\left(j_{t}\right)$, the " \pm " denoting the value of the photoelectron's orbital angular momentum, $l=j_{t} \pm 1$. The parity-unfavored partial cross sections in Eq. (9) depend on the amplitudes $\bar{S}_{0}\left(j_{t}\right)$, the " 0 " denoting $l=j_{t}$. That the asymmetry parameter for any parity-unfavored value of $j_{t}$ is -1 independent of dynamics, as indicated in Eq. (11), is discussed in Ref. 2.

The scattering amplitudes $\bar{S}_{l}\left(j_{t}\right)$ may be expressed as a sum of reduced electric dipole matrix elements, each corresponding to a given total angular momentum $\overrightarrow{\mathrm{J}}^{3}$ :

$$
\begin{aligned}
\bar{S}_{l}\left(j_{t}\right) \equiv & \left(\left(J_{c} s\right) J_{c s} l\left|\bar{S}\left(j_{t}\right)\right| \alpha_{0} J_{0} j_{\gamma}=1\right) \\
= & n(\chi) \sum_{J}(-1)^{J_{0}-J-1} \hat{J}\left\{\begin{array}{ccc}
J_{c s} & l & J \\
1 & J_{0} & j_{t}
\end{array}\right\} \\
& \quad\left(\left(\left(J_{c} s\right) J_{c s} l, J-\left\|P^{[1]}\right\| \alpha_{0} J_{0}\right) .\right.
\end{aligned}
$$

Here, $n(x) \equiv 4 \pi \alpha \hbar \omega / 3 \chi^{2}, \hat{J} \equiv(2 J+1)^{1 / 2}, \alpha_{0}$ denotes the set of quantum numbers necessary to uniquely 
specify the initial state, and the minus sign indicates that the final state is normalized according to incoming-wave boundary conditions. In Eq. (12) the " \pm " and " 0 " subscripts of the scattering-matrix amplitudes are replaced by the appropriate value of $l$. As it stands, Eq. (12) is completely general. The $L S$-coupling form of $\bar{S}_{l}\left(j_{t}\right)$ in terms of reduced electric dipole matrix elements is derived in Sec. III.

Before continuing with this formal development, however, we discuss in the rest of this section the physical significance of the angular momentum transfer and, in particular, its role as a probe of anisotropic electron-ion interactions.

The physical significance of angular momentum transfer as a direct probe of anisotropic electronion interactions is illustrated in Fig. 1. In this analysis it is convenient to think of the photoionization process as having two stages, namely, an initial stage $A$ of photoabsorption proper and a subsequent stage $B$ of escape of the photoelectron from the rest of the atom. The angular momentum transfer is always equal to the difference between the angular momentum input to the atom (namely, the angular momentum $j_{\gamma}=1$ of the electric dipole interaction) and the angular momentum output from the atom (namely, the photoelectron's final-state orbital momentum $\overrightarrow{1}$ ). Thus the angular momentum transfer, $\vec{j}_{t}=\vec{j}_{\gamma}-\vec{i}$, is the net angular momentum transferred to (or deposited in) the target by the photoionization process. (Note that since we consider experiments in which the photoelectron's spin $\overrightarrow{\mathrm{s}}$ is not measured, $\overrightarrow{\mathrm{s}}$ is included as part of the angular momentum of the residual target.) The allowed values of $j_{t}$, however, are different in the two stages of the photoionization process.

In the initial stage $A$ [illustrated in Fig. 1(a)] the photoabsorption imparts $j_{\gamma}=1$ unit of orbital momentum to the photoelectron, which has initial orbital momentum $\overrightarrow{\mathrm{l}}_{0}$ (in an independent-particle model), yielding a final orbital momentum $\overrightarrow{\mathrm{l}}^{\prime}=\overrightarrow{\mathrm{l}}_{0}+\overrightarrow{\mathrm{j}}_{\gamma}$. Therefore in stage $A$ the angular momentum transferred to the target is

$$
\overrightarrow{\mathrm{j}}_{t}^{\prime} \equiv \overrightarrow{\mathrm{j}}_{\gamma}-\overrightarrow{\mathrm{l}}^{\prime}=-\overrightarrow{\mathrm{l}}_{0} \text {, }
$$

where the magnitude $j_{t}^{\prime}$ has the single value $j_{t}^{\prime}=l_{0}$. Furthermore, owing to parity conservation, $l^{\prime}$ $=l_{0} \pm 1$, and hence $j_{t}^{\prime}=l_{0}$ is a parity-favored angular momentum transfer.

During the subsequent escape of the photoelectron in stage $B$ additional angular momentum transfers can arise, within the allowed range determined by Eq. (2), from anisotropic interactions of the photoelectron with the rest of the target. In this report we consider only spin-independent interactions in $L S$ coupling. Therefore the interaction in stage $B$ is that between the orbital motion of the photoelectron and the net orbital motion of the electrons of the residual ion core, as illustrated in Fig. 1(b). This interaction produces a dynamical coupling of the respective orbital momenta $\overrightarrow{1}^{\prime}$ and $\overrightarrow{\mathrm{L}}_{c}$. Owing to the resulting angular momentum exchanges $\overrightarrow{\mathrm{k}}$ between the photoelectron and the core, only the total angular momentum $\overrightarrow{\mathrm{L}}$ is conserved. (It is because of this dependence on $L$ that we call these interactions anisotropic.) In particular, the photoelectron orbital momentum can change from $\overrightarrow{1}^{\prime}$ to $\overrightarrow{1}$ during the departure of the photoelectron from the atom, in which case the angular momentum transfer is no longer $j_{t}^{\prime}=-l_{0}$ but

$$
\overrightarrow{\mathrm{j}}_{t} \equiv \overrightarrow{\mathrm{j}}_{\gamma}-\overrightarrow{\mathrm{l}}=\overrightarrow{\mathrm{j}}_{t}^{\prime}-\overrightarrow{\mathrm{k}}
$$

as illustrated in Fig. 1(c). Note that even if the magnitudes of $\overrightarrow{\mathrm{I}}^{\prime}$ and $\overrightarrow{\mathrm{L}}_{c}^{\prime}$ remain unchanged, a precession (albeit quantized in units of $k$ ) about the total orbital momentum $\overrightarrow{\mathrm{L}}$ is sufficient to produce a change in the magnitude of $\vec{j}_{t}$.

It is at this point that the connection between the present formulation and that of the Cooper-Zare independent-particle model ${ }^{4}$ emerges most clearly. The Cooper-Zare model treats the residual ion core as a spectator to the photoionization process. That is, stage $B$ is ignored altogether, in which case only the single (parity-favored) angular momentum transfer $j_{t}=l_{0}$ arises. In addition, the amplitudes $\bar{S}_{ \pm}\left(j_{t}=l_{0}\right)$ assume limiting forms [cf. Eq. (35) below] which, when substituted in Eq. (10), give the Cooper-Zare formula for the asymmetry parameter. These points are developed in detail in the following sections.

\section{PHOTOIONIZATION AMPLITUDES $\bar{S}_{l}\left(j_{t}\right)$ IN $L S$ COUPLING}

Our task in this section is to obtain the LScoupling form of the reduced electric dipole matrix element in Eq. (12). Before specializing to LS coupling, however, let us consider the problem in general. The form of the reduced dipole matrix element in Eq. (12) is inconvenient for numerical

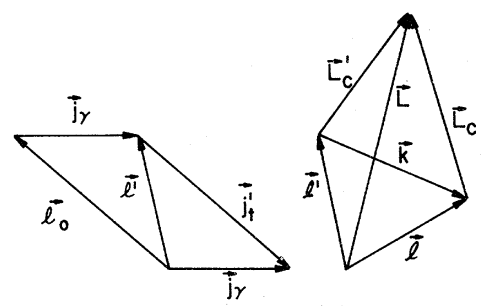

(a)

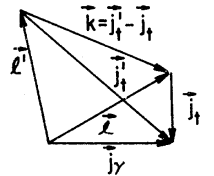

(c)
FIG. 1. Illustration of the origin of multiple angular momentum transfers in atomic photoionization reactions. See text for discussion. 
calculation for two reasons. First, the final state $\left(\left(J_{c} s\right) J_{c s} l, J-\mid\right.$ is defined in terms of the dissociation-channel quantum numbers appropriate to the electron-ion system at infinite separation. In general it is more convenient to calculate the electric dipole matrix element for transition to one of the electron-ion eigenchannel states $(\alpha J)$, where $\alpha$ denotes the eigenchannel coupling scheme. Second, it is much more convenient to calculate real matrix elements, and for this reason a transformation to the standing-wave representation is desirable.

For these reasons, we expand the dipole matrix element in Eq. (12) as follows ${ }^{3}$ :

$$
\begin{aligned}
& \left(\left(J_{c} s\right) J_{c s} l, J-\left\|P^{[1]}\right\| \alpha_{0} J_{0}\right) \\
& \quad=i^{-l} e^{i \delta\left(J_{c} l\right)} \sum_{\alpha}\left(\left(J_{c} s\right) J_{c s} l \mid \alpha\right)^{J} e^{i \delta(\alpha)}\left(\alpha J\left\|P^{[1]}\right\| \alpha_{0} J_{0}\right) .
\end{aligned}
$$

Each term in the summation in Eq. (15) comprises three elements:

(i) The phase factor $i^{-l} e^{i\left[\sigma\left(J_{c} l\right)+\delta(\alpha)\right]}$, which effects the change from incoming-wave to standingwave normalization. Here $\sigma\left(J_{c} l\right)$ is the Coulomb phase,

$$
\sigma\left(J_{c} l\right)=\arg \Gamma(l+1-i / \sqrt{\epsilon}),
$$

which depends on the binding energy $I\left(J_{c}\right)$ of the residual ion fine-structure level $J_{c}$ through the photoelectron kinetic energy $\epsilon$ measured in Rydbergs:

$$
\epsilon=\hbar \omega-I\left(J_{c}\right) .
$$

The phase $\delta(\alpha)$ is the photoelectron phase shift with respect to Coulomb waves in the eigenchannel $\alpha$ and represents the effect of short-range electron-ion interactions.

(ii) The real transformation coefficients $\left(\left(J_{c} s\right) J_{c s} l \mid \alpha\right)^{J}$ which relate the eigenchannel coupling scheme to the dissociation channel coupling scheme.

(iii) The real reduced dipole matrix elements ( $\left.\alpha J\left\|P^{[1]}\right\| \alpha_{0} J_{0}\right)$.

Thus far Eq. (15) and all preceding equations are exact for electric dipole transitions. Approximations must be made, however, in the representation of the eigenchannels $(\alpha J \mid$ and their phase shifts $\delta(\alpha)$ as well as in the representation of the initial state $\left(\alpha_{0} J_{0}\right)$. We proceed in the rest of this section to derive the LS-coupling form of Eq. (15) and then to reduce that further by assuming the use of radial one-electron wave functions appropriate for given term levels of the ion core and the electron-ion system.

In $L S$ coupling $\alpha_{0}$ and $\alpha$ are given by

$$
\alpha_{0}=L_{0} S_{0}, \quad \alpha=\left(L_{c} l\right) L\left(S_{c} s\right) S .
$$

Implicit in the definition of $\alpha_{0}$ is that we have an atomic configuration having a single open shell $l_{0}^{N}$, where $l_{0}$ is the orbital angular momentum and $N$ is the occupation number. Similarly $\alpha$ implicitly indicates the configuration of the final state after photoionization, which is of the form $l_{0}^{N-1} l$. The transformation coefficient in Eq. (15) may be found either algebraically ${ }^{16}$ or graphically ${ }^{17}$ to be

$$
\begin{aligned}
&\left(\left(L_{c} S_{c}\right) J_{c s} l \mid\left(L_{c} l\right) L\left(S_{c} s\right) S\right)^{J} \\
&=(-1)^{2 J} c s^{+\left(L_{c}+l+L\right)+\left(s_{c}+s+s\right)} \hat{J}_{c} \hat{J}_{c s} \hat{L} \hat{S} \\
& \times\left\{\begin{array}{ccc}
L_{c} & S_{c} & J_{c} \\
s & J_{c s} & S
\end{array}\right\}\left\{\begin{array}{lll}
L & S & J \\
J_{c s} & l & L_{c}
\end{array}\right\} \cdot(19)
\end{aligned}
$$

Finally, we must evaluate the $L S$-coupling form of the reduced dipole matrix element

$\left(\alpha J\left\|P^{[1]}\right\| \alpha_{0} J_{0}\right)=\left(\left(L_{c} l\right) L\left(S_{c} s\right) S, J\left\|P^{[1]}\right\| L_{0} S_{0}, J_{0}\right)$.

This evaluation may be carried out graphically, ${ }^{18}$ but in what follows we shall proceed algebraically.

The first step in the evaluation of Eq. (20) is to make a fractional parentage expansion of the initial state,

$$
\begin{aligned}
\left.\left.\mid L_{0} S_{0}, J_{0}\right)=\sum_{\bar{L}_{0} \bar{S}_{0}} \mid\left(\bar{L}_{0} l_{0}\right) L_{0}\left(\bar{S}_{0} s\right) S_{0}, J_{0}\right) \\
\quad \times\left(l_{0}^{N-1} \bar{L}_{0} \bar{S}_{0}, l_{0} \rrbracket l_{0}^{N} L_{0} S_{0}\right) .
\end{aligned}
$$

Since the ionization process is spin independent, the second step is to split off the geometrical dependence of the matrix element in Eq. (20) on spin and total angular momentum quantum number $\mathrm{s}^{19}$ :

$$
\begin{aligned}
\left(\left(L_{c} l\right) L\left(S_{c} s\right) S J\left\|P^{[1]}\right\|\left(\bar{L}_{0} l_{0}\right) L_{0}\left(\bar{S}_{0} s\right) S_{0}, J_{0}\right) \\
=(-1)^{L+S+J_{0}+1} \hat{J} \hat{J}_{0}\left\{\begin{array}{ccc}
J & J_{0} & 1 \\
L_{0} & L & S_{0}
\end{array}\right\} \delta\left(S_{c}, \bar{S}_{0}\right) \delta\left(S, S_{0}\right) \\
\quad \times\left(\left(L_{c} l\right) L\left\|P^{[1]}\right\|\left(\bar{L}_{0} l_{0}\right) L_{0}\right) .
\end{aligned}
$$

The third step is to reduce the matrix element of the electric dipole operator to a one-electron matrix element by factoring out the geometrical dependence on core and total orbital momenta ${ }^{20}$ :

$$
\begin{aligned}
& \left(\left(L_{c} l\right) L\left\|P^{[1]}\right\|\left(\bar{L}_{0} l_{0}\right) L_{0}\right) \\
& =N^{1 / 2}(-1)^{L_{c}+l_{0}+L+1} \hat{L} \hat{L}_{0}\left\{\begin{array}{ccc}
L & L_{0} & 1 \\
l_{0} & l & L_{c}
\end{array}\right\} \\
& \\
& \quad \times \delta\left(L_{c}, \bar{L}_{0}\right)\left(l\left\|P^{[1]}\right\| l_{0}\right) .
\end{aligned}
$$

In this equation the factor $N^{1 / 2}$ is a weight factor due to the presence of $N$ equivalent electrons in the initial state. ${ }^{18,21}$ The last step is to factor the reduced one-electron matrix element into its radial and angular parts ${ }^{22}$ : 


$$
\left(l\left\|P^{[1]}\right\| l_{0}\right)=\left(l\left\|C^{[1]}\right\| l_{0}\right) R_{\epsilon l}^{L} c_{c} s_{c}^{L},
$$

where the angular part is

$$
\left(l\left\|C^{[1]}\right\| l_{0}\right)=(-1)^{l} \widehat{l}_{0}\left(\begin{array}{ccc}
l & 1 & l_{0} \\
0 & 0 & 0
\end{array}\right)
$$

and the radial part is

$$
R_{\epsilon l}^{L_{c} S_{c} L} \equiv \int_{0}^{\infty} d r \psi_{f}\left(\left(L_{c} S_{c}\right) \epsilon l, L S \mid r\right) r \psi_{i}\left(n_{0} l_{0}, L_{0} S_{0} \mid r\right) .
$$

Note particularly that this radial part is calculated using radial wave functions dependent dynamically on the angular momentum and spin quantum numbers of the initial state, of the final state, and of the residual ion core.

Putting Eqs. (21)-(26) together, we find for the reduced dipole matrix element in Eq. (20)

$$
\begin{aligned}
& \left(\left(L_{c} l\right) L\left(S_{c} s\right) S, J\left\|P^{[1]}\right\| L_{0} S_{0}, J_{0}\right) \\
& =N^{1 / 2}\left(l_{0}^{N-1} L_{c} S_{c}, l_{0} \mathbb{l} l_{0}^{N} L_{0} S_{0}\right)(-1)^{L_{c}+S_{0}+J_{0}+1} \hat{J}_{J_{0}} \hat{L} \hat{L}_{0} \hat{l}_{0}\left\{\begin{array}{ccc}
J & J_{0} & 1 \\
L_{0} & L & S
\end{array}\right\}\left\{\begin{array}{ccc}
L & L_{0} & 1 \\
l_{0} & l & L_{c}
\end{array}\right\}\left(\begin{array}{lll}
l & 1 & l_{0} \\
0 & 0 & 0
\end{array}\right) R_{\epsilon l}^{L_{c} s_{c} L} .
\end{aligned}
$$

Finally, substituting Eqs. (15), (19), (20), and (27) into Eq. (14) and noting that $(-1)^{J_{0}-J}=(-1)^{J-J_{0}}$, we find for the scattering amplitude the following result:

$$
\begin{aligned}
& \bar{S}_{l}\left(j_{t}\right) \equiv n(\chi) N^{1 / 2}\left(l_{0}^{N-1} L_{c} S_{c}, l_{0} \rrbracket l_{0}^{N} L_{0} S_{0}\right) i^{-l} e^{i \sigma\left(J_{c} l\right)}(-1)^{s_{c}+s+S_{0}} \tilde{l l}_{0}\left(\begin{array}{ccc}
l & 1 & l_{0} \\
0 & 0 & 0
\end{array}\right) \hat{J}_{0} \hat{J}_{c} \hat{J}_{c s} \hat{L}_{0} \hat{S}_{0}\left\{\begin{array}{ccc}
L_{c} & S_{c} & J_{c} \\
s & J_{c s} & S_{0}
\end{array}\right\} \\
& \times \sum_{L} e^{i \delta_{\epsilon l}^{L_{c} s_{c} L}{ }_{R}^{L_{c l} s_{c} L}} \hat{L}^{2}\left\{\begin{array}{ccc}
L_{0} & L_{c} & l_{0} \\
l & 1 & L
\end{array}\right\}(-1)^{2 J_{c s^{+}} s_{0}+l+L} \sum_{J}(-1)^{J} \hat{J}^{2}\left\{\begin{array}{ccc}
J_{c s} & J & l \\
1 & j_{t} & J_{0}
\end{array}\right\}\left\{\begin{array}{lll}
J_{0} & J & 1 \\
L & L_{0} & S_{0}
\end{array}\right\}\left\{\begin{array}{lll}
S_{0} & J & 1 \\
l & L_{c} & J_{c s}
\end{array}\right\} .
\end{aligned}
$$

Since we have assumed no dependence of the phase shifts and radial dipole matrix elements on total angular momentum $J$, the sum over $J$ in Eq. (28) may be performed analytically using the Biedenharn identity ${ }^{23}$ to yield the desired expression for the scattering amplitude in $L S$ coupling:

$$
\begin{aligned}
\bar{S}_{l}\left(j_{t}\right)= & n(\chi) N^{1 / 2}\left(l_{0}^{N-1} L_{c} S_{c}, l_{0} \rrbracket l_{0}^{N} L_{0} S_{0}\right) i^{-l} e^{i \sigma\left(J_{c} l\right)} Q\left(j_{t}, J_{c}, J_{c s}\right)(-1)^{\left(L_{0}+L_{c}+1\right)+\left(S_{c}+s+S_{0}\right)+\left(J_{c s}-J_{0}-j_{t}\right)} \hat{J}_{0} \tilde{l}_{0}\left(\begin{array}{lll}
l & 1 & l_{0} \\
0 & 0 & 0
\end{array}\right) \\
& \times \sum_{L} e^{i \delta_{\epsilon l}^{L_{c} S_{c} L} R_{\epsilon l}^{L_{c} S_{c} L}} \hat{L}^{2}\left\{\begin{array}{ccc}
L_{0} & L_{c} & j_{t} \\
l & 1 & L
\end{array}\right\}\left\{\begin{array}{ccc}
L_{0} & L_{c} & l_{0} \\
l & 1 & L
\end{array}\right\},
\end{aligned}
$$

where

$$
Q\left(j_{t}, J_{c}, J_{c s}\right) \equiv \hat{L}_{0} \hat{S}_{0} \hat{J}_{c} \hat{J}_{c s}\left\{\begin{array}{lll}
J_{c} & L_{c} & S_{c} \\
S_{0} & s & J_{c s}
\end{array}\right\}\left\{\begin{array}{lll}
j_{t} & L_{c} & L_{0} \\
S_{0} & J_{0} & J_{c s}
\end{array}\right\} .
$$

Though Eq. (29) gives the $L S$-coupling form of the scattering amplitude, we see that there is a geometrical dependence on the quantum numbers $J_{c s}$ and $J_{c}$ relating to the fine-structure levels of the ionic core. (We have neglected any dependence of the phase shifts $\delta_{\epsilon l}^{L_{C} S_{C} L}$ and radial dipole matrix elements $R_{\epsilon l}^{L_{c} S_{c} L}$ on the fine-structure levels.) We consider this dependence on $J_{c s}$ and $J_{c}$ in turn.

All of the dependence on $J_{c s}$ in Eq. (29) is contained in the geometrical factor $(-1)^{J_{C S}-J_{0}}$ $\times Q\left(j_{t}, J_{c}, J_{c s}\right)$, which depends additionally on quantum numbers that are either fixed for a given ionization process (e.g., $s, L_{0}, S_{0}, L_{c}, S_{c}, J_{c}$ ) or enter incoherently in the differential and total cross sections (e.g., $j_{t}$ in addition to $J_{c s}$ ). The square of this factor, with phase +1 , enters into the definitions of the cross section [Eq. (6)] and the asymmetry parameter [Eq. (7)], each of which involves a summation over $J_{c s}$. Accordingly, it is convenient to define a new quantity

$$
\bar{Q}\left(j_{t}, J_{c}\right)^{2} \equiv \sum_{J_{c s}} Q\left(j_{t}, J_{c}, J_{c s}\right)^{2}
$$

which gives the statistical weight with which ionization probability for a given $j_{t}$ is distributed 
among the possible fine-structure levels $J_{c}$, since

$$
\sum_{J_{c}} \bar{Q}\left(j_{t}, J_{c}\right)^{2}=1
$$

Note, however, that in Eq. (29) there is an additional dynamical dependence on $J_{c}$ arising from the Coulomb phase $\sigma\left(J_{c} l\right)$. Often, though, the finestructure separations of the residual ion are not resolved. Then $\sigma\left(J_{c} l\right)$ can be taken as independent of $J_{c}$, and the dependence of the cross sections and asymmetry parameter on $J_{c}$ can be removed altogether by application of Eq. (32).

Having obtained the form of the scattering amplitude in $L S$ coupling in Eq. (29), it is instructive to return to our discussion in the last section concerning the role of $j_{t}$ as a probe of anisotropic interactions, as illustrated in Fig. 1. The allowed values of $j_{t}$ are those consistent with the triangular relations $\left\{L_{0} L_{c} j_{t}\right\}$ and $\left\{l 1 j_{t}\right\}$, implied by the first $6 j$ symbol in Eq. (29). The coupling of the electron to the residual ion [cf. Fig. 1(b)] is reflected in the dependence of the phase shifts $\delta_{\epsilon l}^{L_{C} S_{c} L}$ and dipole matrix elements $R_{\epsilon l}^{L_{c} S_{c} L}$ on the total angular momentum of the electron-ion complex. Only when these phases and matrix elements do not depend on $L$ (i.e., when the electron-ion interaction is isotropic) is $j_{t}$ restricted to the single value $j_{t}=l_{0}$. For in this case the dynamical weight factors in Eq. (29) may be extracted from the summation, since

$$
e^{i \delta_{\epsilon l}^{L_{C} S_{C} L} R_{\epsilon l}^{L_{C} S_{C} L}} \frac{\text { isotropic }}{\text { interaction }} e^{i \delta_{\epsilon l} R_{\epsilon l}},
$$

and the summation over $L$ may be performed analytically:

$$
\sum_{L} \hat{L}^{2}\left\{\begin{array}{ccc}
L_{0} & L_{c} & j_{t} \\
l & 1 & L
\end{array}\right\}\left\{\begin{array}{ccc}
L_{0} & L_{c} & l_{0} \\
l & 1 & L
\end{array}\right\}=\hat{l}_{0}^{-2} \delta\left(j_{t}, l_{0}\right) \text {. }
$$

The scattering amplitude then depends only on the final orbital angular momentum $l$ of the photoelectron,

$$
\bar{S}_{l}\left(j_{t}=l_{0}\right) \propto i^{-l} e^{i\left(\sigma_{\epsilon l}+\delta_{\epsilon l}\right)} \hat{l}_{0}\left(\begin{array}{ccc}
l & 1 & l_{0} \\
0 & 0 & 0
\end{array}\right) R_{\epsilon l},
$$

where the proportionality constant indicates that we have not written down all the other factors from Eq. (29) which depend on quantum numbers that are fixed for a given photoionization process. These other factors do not contribute to the asymmetry parameter in Eq. (10) since they occur in both numerator and denominator and thus cancel out. Setting $j_{t}=l_{0}$ in Eq. (10) and substituting the scattering amplitude from Eq. (35) leads to the asym- metry parameter $\beta\left(l_{0}\right)$ of the Cooper-Zare independent-particle model. ${ }^{4}$

\section{APPLICATION TO SULFUR PHOTOIONIZATION}

To illustrate the theory developed in the last two sections we calculate the angular distribution of photoelectrons ionized from atomic sulfur according to the reaction

$$
S\left(3 p^{4}{ }^{3} P\right)+\gamma \rightarrow S^{+}\left(3 p^{3}{ }^{4} S,{ }^{2} D,{ }^{2} P\right)+e^{-} .
$$

For each of the residual ion terms $L_{c} S_{c}$ we present in Table I the allowed values of photoelectron angular momentum $l$, angular momentum transfer $j_{t}$, reaction parity (where parity change $=+1$ is favored and parity change $=-1$ is unfavored), and the allowed values of total angular and spin momenta for the electron-ion system. We see that the ${ }^{4} S$-ion term has only the single angular momentum transfer $j_{t}=l_{0}=1$, but that the ${ }^{2} D$ and ${ }^{2} P$ ion terms both have other values of $j_{t}$ including parityunfavored values. Note that for the ${ }^{2} D$ - and ${ }^{2} P$-ion terms the $l=2$ states have two or more allowed values of $L S$, implying that there will be interference between phase shifts belonging to different final-state channels.

It should be noted that the values of the quantum numbers appearing in Table I are all those obtained from Eqs. (2)-(4) that are consistent with our approximations, given in Sec. III. In particular, angular momentum and parity conservation, represented by Eqs. (2)-(4), allow the photoelectron orbital angular momentum $l$ to have the values $0,2,4$, and 6 for the reaction

$$
S\left(3 p^{4} J_{0}=2\right)+h \nu \rightarrow S^{+}\left(3 p^{3} J_{0}=\frac{5}{2}\right)+e^{-}(l) .
$$

However, in our $L S$-coupling single-configuration approximation for the ground state the electric dipole operator restricts the photoelectron to $l=0$, 2 [cf. Eq. (25)]. One may obtain $l=4$ photoelectrons in $L S$ coupling by considering the interaction of the ground $3 p^{4}$ configuration with other highly excited configurations, as found by Conneely, Smith, and Lipsky. ${ }^{24}$ To obtain $l=6$ photoelectrons one must either abandon $L S$ coupling and consider relativistic interactions for which only the total angular momentum is conserved, or else, keep $L S$ coupling but consider the photoionization of highly excited ground states to excited ion states that do not interact with the configuration $3 p^{3}$. This promotion of the photoelectron to $l=4$ or $l=6$ is a specific example of anisotropic interaction as represented schematically in Fig. 1(b).

For conciseness we shall concentrate in what follows on the photoionization reaction leading to the ${ }^{2} D$-ion term since it shows the strongest anisotropic electron-ion interactions. For this ion 
term the scattering amplitudes $\bar{S}_{l}\left(j_{t}\right)$ in Eq. (29) for the allowed values of $l$ and $j_{t}$ listed in Table I are

$$
\begin{aligned}
& \bar{S}_{s}(1)=C \frac{1}{3} e^{i\left[\sigma_{s}+\delta_{s}\left({ }^{3} D\right)\right]} R_{\epsilon s}\left({ }^{3} D\right),
\end{aligned}
$$

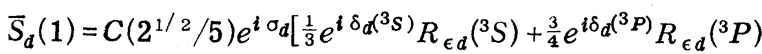

$$
\begin{aligned}
& \left.+\frac{7}{12} e^{i \delta_{d}^{(3 D)}} R_{\epsilon d}\left({ }^{3} D\right)\right],
\end{aligned}
$$

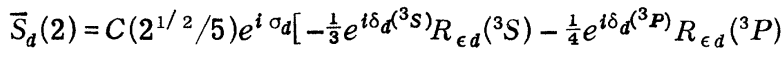

$$
\begin{aligned}
& \left.+\frac{7}{12} e^{i \delta_{d}\left({ }^{3} D\right)} R_{\epsilon d}\left({ }^{3} D\right)\right],
\end{aligned}
$$

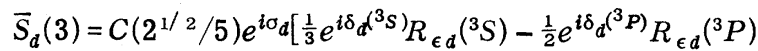

$$
\begin{aligned}
& \left.+\frac{1}{6} e^{i \delta_{d}\left({ }^{3} D\right)} R_{\epsilon d}\left({ }^{3} D\right)\right] \text {. }
\end{aligned}
$$

In Eq. (37) $C$ denotes those constant factors in Eq. (29) that are common to all channels, $s$ and $d$ denote $l=0$ and $l=2$, and $R_{\epsilon d}\left({ }^{3} S\right)$, for example, denotes the radial dipole matrix element $R_{\epsilon l}^{L_{c} S_{c} L}$ for $L_{c} S_{c}={ }^{2} D, L=0$, and $l=2$. Note that we have ig-
TABLE I. Allowed values for the ion-core term level $L_{c} S_{c}$, photoelectron orbital angular momentum $l$, angular momentum transfer $j_{t}$, reaction parity $\pi_{0} \pi_{c}(-1)^{j_{t}}$, and total orbital and spin angular momenta $L S$ for the reaction $S\left(3 p^{4}{ }^{3} P\right)+h \nu \rightarrow S^{+}\left(3 p^{3}{ }^{4} S,{ }^{2} D,{ }^{2} P\right)+e^{-}$.

\begin{tabular}{lllll}
\hline \hline$L_{c} S_{c}$ & $l$ & $j_{t}$ & Parity & \multicolumn{1}{c}{$L S$} \\
\hline${ }^{4} S$ & 0 & 1 & +1 (fav) & ${ }^{3} S$ \\
${ }^{4} S$ & 2 & 1 & +1 (fav) & ${ }^{3} D$ \\
${ }^{2} D$ & 0 & 1 & +1 (fav) & ${ }^{3} D$ \\
${ }^{2} D$ & 2 & 1 & +1 (fav) & ${ }^{3} D,{ }^{3} P,{ }^{3} S$ \\
${ }^{2} D$ & 2 & 2 & -1 (unf) & ${ }^{3} D,{ }^{3} P,{ }^{3} S$ \\
${ }^{2} D$ & 2 & 3 & +1 (fav) & ${ }^{3} D,{ }^{3} P,{ }^{3} S$ \\
${ }^{2} P$ & 0 & 1 & +1 (fav) & ${ }^{3} P$ \\
${ }^{2} P$ & 2 & 1 & +1 (fav) & ${ }^{3} D,{ }^{3} P$ \\
${ }^{2} P$ & 2 & 2 & -1 (unf) & ${ }^{3} D,{ }^{3} P$ \\
\hline \hline
\end{tabular}

nored the dependence of the Coulomb phase shifts $\sigma_{s}$ and $\sigma_{d}$ on ion-core fine-structure levels $J_{c}$, as discussed at the end of the previous section.

The asymmetry parameter for photoionization to the ${ }^{2} D$-ion term is given by Eqs. (7)-(11):

$$
\beta=\frac{3\left|\bar{S}_{d}(1)\right|^{2}-3 \times 2^{1 / 2}\left[\bar{S}_{d}(1) \bar{S}_{s}(1)^{\dagger}+\text { c.c. }\right]-5\left|\bar{S}_{d}(2)\right|^{2}+2\left|\bar{S}_{d}(3)\right|^{2}}{3\left|S_{s}(1)\right|^{2}+3\left|\bar{S}_{d}(1)\right|^{2}+5\left|\bar{S}_{d}(2)\right|^{2}+7\left|\bar{S}_{d}(3)\right|^{2}} .
$$

In Eq. (38) the common factor $C$ in Eq. (37) cancels in numerator and denominator. As pointed out in the last section, Eq. (38) reduces to the CooperZare result for $\beta^{4}$ when the phase shifts $\delta_{\epsilon l}^{L_{c} S_{c} L}$ and radial dipole matrix elements $R_{\epsilon l}^{L_{c} S_{c} L}$ become independent of $L_{c} S_{c} L$. It is of interest to see how this occurs for this particular example. Note first that $\bar{S}_{d}(2) \rightarrow 0$ and $\bar{S}_{d}(3) \rightarrow 0$ in Eqs. $(37 \mathrm{c})$ and $(37 d)$ when the phase shifts and dipole matrix elements become identical. We also see that the squared modulus of each of these scattering amplitudes, having $j_{t} \neq l_{0}=1$, is nonzero partly because the resulting factors $\cos \left[\delta_{d}\left({ }^{3} S\right)-\delta_{d}\left({ }^{3} P\right)\right]$, etc., in the cross terms are not unity. These same factors also arise in cross terms of $\left|S_{d}(1)\right|^{2}$ and are partly responsible for changing the value of this modulus from what its (nonzero) value would be in a Cooper-Zare model calculation. For these reasons we regard the magnitude of phase-shift differences $\delta_{\epsilon l}^{I_{c} S_{c} L}-\delta_{\epsilon l}^{L_{c} S_{c} L^{\prime}}$ to be an indication of the strength of anisotropic electron-ion interactions and hence of the validity of the Cooper-Zare model for $\beta .^{4}$

The scattering amplitudes in Eqs. (37a) and (37b), having $j_{t}=l_{0}=1$, contribute to the cross section and the asymmetry parameter $\beta$ whether the phase shifts and matrix elements are identical or not. The scattering amplitudes in Eqs. (37c) and (37d), however, having $j_{t} \neq l_{0}=1$, contribute only when the phases and matrix elements are dif- ferent from one another. An index of the strength of angular momentum transfers $j_{t} \neq l_{0}$ is thus the fraction $\left[\sigma-\sigma\left(j_{t}=l_{0}\right)\right] / \sigma$, where $\sigma$ is the photoionization cross section and $\sigma\left(j_{t}=l_{0}\right)$ is the partial cross section corresponding to $j_{t}=l_{0}$. For photoionization to the ${ }^{2} D$ ion term of sulfur this ratio is expressed as

$$
\begin{aligned}
{[\sigma-\sigma(1)] / \sigma=} & {\left[5\left|\bar{S}_{d}(2)\right|^{2}+7\left|\bar{S}_{d}(3)\right|^{2}\right] / } \\
& {\left[3\left|\bar{S}_{s}(1)\right|^{2}+3\left|\bar{S}_{d}(1)\right|^{2}\right.} \\
& \left.+5\left|\bar{S}_{d}(2)\right|^{2}+7\left|\bar{S}_{d}(3)\right|^{2}\right] .
\end{aligned}
$$

To evaluate Eqs. (37)-(39) we used continuum Hartree-Fock (HF) wave functions obtained by solving the equations given by Dalgarno, Henry, and Stewart ${ }^{25}$ using methods discussed fully by Kennedy and Manson. ${ }^{6}$ These continuum wave functions depend on both the ionic term level and the total orbital angular momentum. Discrete HF single-particle orbitals for the neutral atom and for the ion were obtained from the tabulation of Clementi. ${ }^{26}$

For comparison, we have also carried out a Cooper-Zare type of calculation employing Herman-Skillman ${ }^{27}$ (HS) wave functions. The continuum HS wave functions are calculated in the average sulfur potential appropriate to the ground configuration as tabulated by Herman and Skillman. ${ }^{27}$ These wave functions depend neither on the ion 
core level $L_{c} S_{c}$ nor on the total angular momentum $L$, and thus the phase shifts and radial dipole matrix elements depend only on $\epsilon l$, and Eq. (37) reduces to

$$
\begin{aligned}
& \bar{S}_{s}(1)=C \frac{1}{3} e^{i\left(\sigma_{s}+\delta_{s}\right)} R_{\epsilon s}, \\
& \bar{S}_{d}(1)=C\left(2^{1 / 2} / 3\right) e^{i\left(\sigma_{d}+\delta_{d}\right)} R_{\epsilon d}, \\
& \bar{S}_{d}(2)=\bar{S}_{d}(3)=0 .
\end{aligned}
$$

Note that since the HS continuum wave functions do not depend on the ionic term level the asymmetry parameters for each ion term, when plotted versus photoelectron kinetic energy $\epsilon$, are identical. Discrete wave functions for both the ion and the atom were taken to be the tabulated HS neutral-atom discrete wave functions.

In Fig. 2 we have plotted HF phase shifts $\delta_{\epsilon d}^{L_{C} S_{C} L}$ for the ionic term level ${ }^{2} D$ as a function of photoelectron kinetic energy $\epsilon$. The three allowed values of $L$ are listed in Table I. These phase shifts differ by as much as $0.7 \mathrm{rad}$, indicating that anisotropic electron-ion interactions are significantly large.

In Fig. 3 we have plotted the three asymmetry parameters corresponding to the three alternative ionic term levels resulting from photoionization of the sulfur atom. Contrary to the Cooper-Zare model, these asymmetry parameters are significantly different from one another when plotted as a function of photoelectron kinetic energy. In this plot the length formula for electric dipole transitions has been used since this is the correct one for $\mathrm{HF}$ calculations. ${ }^{28}$ In Table II, however, we list calculated $\mathrm{HF}$ asymmetry parameters using both length and velocity formulas for the dipole matrix elements in order to show that for most energies listed the differences between the asymmetry parameters for different ion terms are larger than the length and velocity difference for a given ion term. We also list for comparison the

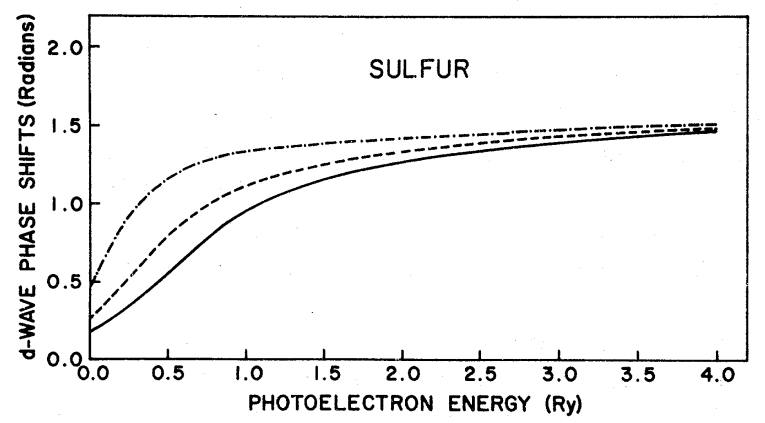

FIG. 2. Hartree-Fock $d$-wave phase shifts $\delta_{\epsilon d}^{L_{c} S_{c}{ }^{L}}$ for the ${ }^{2} D$ sulfur-ion term versus photoelectron kinetic energy $\epsilon$ for alternative allowed values of $L$. Solid line, $L=0$ [i.e., the state $3 p^{3}\left({ }^{2} D\right) \epsilon d^{3} S$; dashed line, $L=1\left({ }^{3} P\right)$; dot-dashed line, $L=2\left({ }^{3} D\right)$.

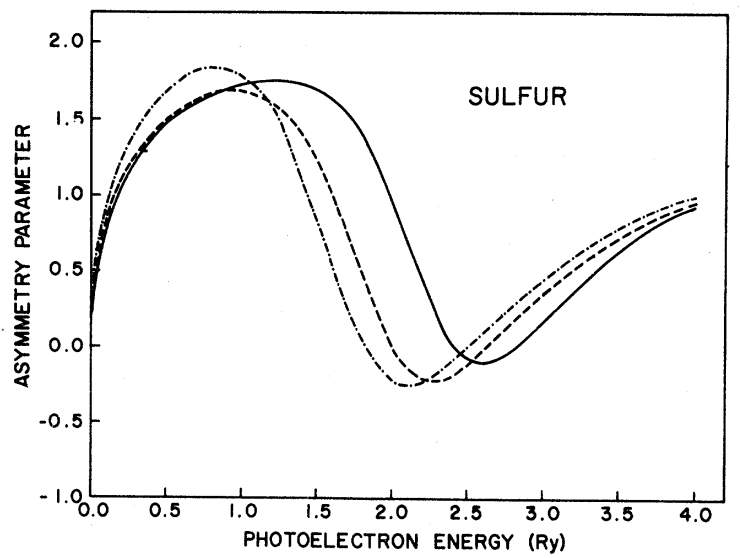

FIG. 3. Asymmetry parameters $\beta\left({ }^{3} P \rightarrow L_{c} S_{c}\right)$ for the photoionization reactions $S\left(3 p^{4}{ }^{3} P\right) \rightarrow S^{+}\left(3 p^{3} L_{c} S_{c}\right)+e^{-}$as a function of photoelectron kinetic energy. Solid line, ${ }^{4} S$ ionic term; dashed line, ${ }^{2} D$; dot-dashed, ${ }^{2} P$.

$\beta$ parameter calculated using HS wave functions and the Cooper-Zare formula for $\beta$ [i.e., Eq. (40)]. The HS wave functions are quite different from the $\mathrm{HF}$ wave functions, and thus the HS asymmetry parameter does not seem to be an "average" of the HF asymmetry parameters at low energies.

In Table III we have plotted HF and HS cross sections for photoionization of sulfur. Note that the HS cross section is a total cross section and would correspond to the sum of the three HF partial cross sections at a given photon energy. However, we have plotted the $\mathrm{HF}$ partial cross sections as functions of photoelectron kinetic energy for comparison with Table II. Comparing Tables II and III, we see that the largest differences in the asymmetry parameters occur for energies 1.5 $\leqslant \epsilon \leqslant 2.1 \mathrm{Ry}$. This is just before the Cooper mini$\mathrm{ma}^{29}$ in the cross sections, which occur in the region $2.1 \leqslant \epsilon \leqslant 2.8 \mathrm{Ry}$. The cross sections in the region $1.5 \leqslant \epsilon \leqslant 2.1 \mathrm{Ry}$ are of the order of $10^{-18} \mathrm{~cm}^{2}$, and thus measurement of $\beta$ for the different thresholds should be experimentally possible, if not for sulfur then for some other element. Simply put, we wish to emphasize that the differences we have found between the asymmetry parameters for the different ionic term levels are not dependent on being at a cross-section minimum. Indeed, as seen in Fig. 3 and Tables II and III there are measurable differences between $\beta\left({ }^{3} P \rightarrow{ }^{4} S\right)$ and $\beta\left({ }^{3} P\right.$ ${ }^{2} P$ ) in the energy range $0.1 \leqslant \epsilon \leqslant 0.8 \mathrm{Ry}$, where the cross sections are of the order of $10^{-17} \mathrm{~cm}^{2}$.

Finally, in Fig. 4 we examine the influence of angular momentum transfers $j_{t} \neq l_{0}$ on the asymmetry parameter and partial cross section for photoionization to the ${ }^{2} D$ ionic term level. The solid line represents the asymmetry parameter given by Eq. (38) and plotted also in Fig. 3. The 
TABLE II. HF asymmetry parameters for the reactions $S\left(3 p^{4}{ }^{3} p\right)+h \nu \rightarrow S^{+}\left(3 p^{3}{ }^{4} S,{ }^{2} D, \quad \boldsymbol{P}\right)$ $+e^{-}$as a function of photoelectron kinetic energy $\epsilon$ using dipole-length (velocity) formula and comparison with HS asymmetry parameter.

\begin{tabular}{rcccr}
\hline \hline$\epsilon$ (Ry) & $\beta\left({ }^{3} P \rightarrow{ }^{4} S\right)$ & $\beta\left({ }^{3} P \rightarrow{ }^{2} D\right)$ & $\beta\left({ }^{3} P \rightarrow{ }^{2} P\right)$ & HS $\beta$ \\
\hline 0.00 & $0.144(0.054)$ & $0.176(0.248)$ & $0.274(0.360)$ & 0.35 \\
0.05 & $0.551(0.495)$ & $0.584(0.614)$ & $0.705(0.739)$ & 0.74 \\
0.10 & $0.769(0.734)$ & $0.805(0.814)$ & $0.942(0.950)$ & 0.96 \\
0.20 & $1.052(1.048)$ & $1.090(1.082)$ & $1.248(1.229)$ & 1.25 \\
0.40 & $1.365(1.407)$ & $1.402(1.402)$ & $1.594(1.564)$ & 1.61 \\
0.60 & $1.543(1.619)$ & $1.574(1.604)$ & $1.779(1.761)$ & 1.78 \\
0.80 & $1.659(1.761)$ & $1.670(1.735)$ & $1.851(1.856)$ & 1.82 \\
1.00 & $1.734(1.849)$ & $1.693(1.782)$ & $1.793(1.820)$ & 1.68 \\
1.25 & $1.772(1.860)$ & $1.581(1.640)$ & $1.472(1.488)$ & $\cdots$ \\
1.50 & $1.714(1.665)$ & $1.234(1.131)$ & $0.847(0.775)$ & 0.10 \\
1.80 & $1.408(0.944)$ & $0.497(0.185)$ & $0.044(-0.078)$ & -0.24 \\
2.10 & $0.724(0.016)$ & $-0.127(-0.270)$ & $-0.250(-0.268)$ & -0.02 \\
2.30 & $0.211(-0.210)$ & $-0.223(-0.207)$ & $-0.173(-0.136)$ & 0.18 \\
2.60 & $-0.108(-0.029)$ & $-0.029(0.076)$ & $0.094(0.155)$ & 0.44 \\
2.80 & $-0.013(0.189)$ & $0.170(0.273)$ & $0.281(0.339)$ & 0.60 \\
3.00 & $0.177(0.393)$ & $0.360(0.447)$ & $0.450(0.500)$ & 0.74 \\
4.00 & $0.933(1.003)$ & $0.972(0.989)$ & $0.996(1.009)$ & 1.10 \\
8.00 & $1.576(1.526)$ & $1.513(1.523)$ & $1.522(1.526)$ & 1.54 \\
15.00 & $1.697(1.629)$ & $1.614(1.636)$ & $1.622(1.636)$ & 1.62 \\
30.00 & $1.582(1.561)$ & $1.534(1.564)$ & $1.537(1.564)$ & 1.54 \\
\hline \hline
\end{tabular}

dashed line represents $\beta$ calculated according to Eq. (38), but setting $\bar{S}_{d}(2)=\bar{S}_{d}(3)=0$. Note that the result is not the Cooper-Zare expression for $\beta$ since we still use Eq. (37b) for $\vec{S}_{d}(1)$, and thus the dependence of phase shifts and radial dipole ma-

TABLE III. HF cross sections for the reactions $S\left(3 p^{4}{ }^{3} P\right)+h \nu \rightarrow S^{+}\left(3 p^{34} S,{ }^{2} D,{ }^{2} \boldsymbol{P}\right)+e^{-}$as a function of photoelectron kinetic energy $\epsilon$ using dipole length (velocity) formula and comparison with HS cross section. All cross sections are in units of $10^{-18} \mathrm{~cm}^{2}$.

\begin{tabular}{rcccc}
\hline \hline$\epsilon$ (Ry) & $\sigma\left({ }^{3} P \rightarrow{ }^{4} S\right)$ & $\sigma\left({ }^{3} P \rightarrow{ }^{2} D\right)$ & $\sigma\left({ }^{3} P \rightarrow{ }^{2} P\right)$ & $\sigma(\mathrm{HS})$ \\
\hline 0.00 & $13.82(8.92)$ & $27.43(19.23)$ & $20.87(15.40)$ & 58.00 \\
0.05 & $14.61(9.11)$ & $28.00(19.18)$ & $20.78(15.04)$ & 58.78 \\
0.10 & $15.03(9.10)$ & $27.46(18.42)$ & $19.73(14.04)$ & 57.24 \\
0.20 & $15.04(8.65)$ & $24.21(15.65)$ & $16.03(11.09)$ & 48.33 \\
0.40 & $12.85(6.85)$ & $15.42(9.38)$ & $8.43(5.62)$ & 24.60 \\
0.60 & $9.47(4.75)$ & $8.70(5.05)$ & $4.01(2.62)$ & 11.84 \\
0.80 & $6.27(2.97)$ & $4.67(2.61)$ & $1.92(1.23)$ & 4.73 \\
1.00 & $3.84(1.73)$ & $2.47(1.34)$ & $0.95(0.61)$ & 2.00 \\
1.25 & $1.96(0.82)$ & $1.14(0.59)$ & $0.44(0.27)$ & $\cdots$ \\
1.50 & $0.97(0.38)$ & $0.56(0.29)$ & $0.23(0.15)$ & 0.58 \\
1.80 & $0.42(0.17)$ & $0.29(0.17)$ & $0.15(0.10)$ & 0.52 \\
2.10 & $0.21(0.11)$ & $0.20(0.15)$ & $0.13(0.10)$ & 0.57 \\
2.30 & $0.16(0.10)$ & $0.19(0.16)$ & $0.13(0.10)$ & 0.61 \\
2.60 & $0.14(0.12)$ & $0.20(0.18)$ & $0.14(0.12)$ & 0.68 \\
2.80 & $0.14(0.13)$ & $0.21(0.19)$ & $0.15(0.12)$ & 0.71 \\
3.00 & $0.15(0.14)$ & $0.22(0.20)$ & $0.15(0.13)$ & 0.75 \\
4.00 & $0.22(0.17)$ & $0.27(0.23)$ & $0.17(0.14)$ & 0.80 \\
8.00 & $0.17(0.12)$ & $0.18(0.15)$ & $0.11(0.09)$ & 0.42 \\
15.00 & $0.05(0.04)$ & $0.06(0.05)$ & $0.04(0.03)$ & 0.15 \\
30.00 & $0.01(0.01)$ & $0.01(0.01)$ & $0.01(0.01)$ & 0.03 \\
\hline \hline
\end{tabular}

trix elements on $L_{c} S_{c} L$ is still important. We see from Fig. 4 that values of $j_{t} \neq l_{0}=1$ lower $\beta$ as much as 0.2 units in the neighborhood of $\epsilon \simeq 1.0 \mathrm{Ry}$. The dot-dashed curve is a plot of the ratio $[\sigma-\sigma(1)] / \sigma$ given in Eq. (39). Values of $j_{t} \neq l_{0}=1$ contribute as much as $8 \%$ to the partial cross section in the neighborhood of $\epsilon \simeq 1.5 \mathrm{Ry}$.

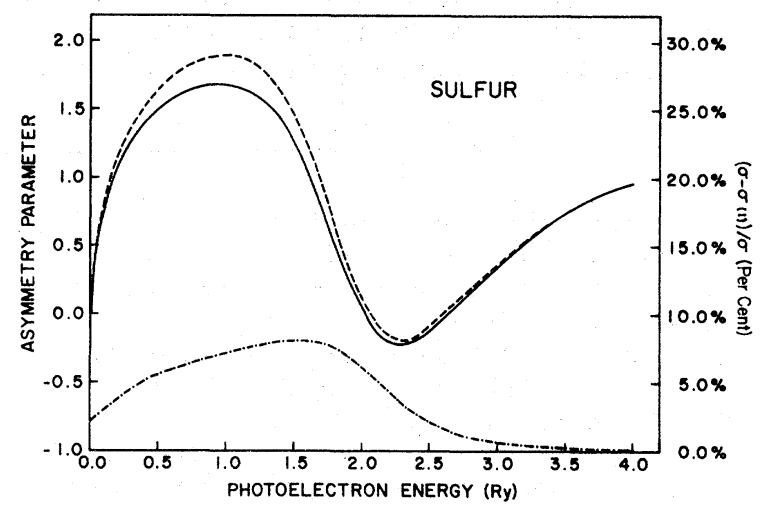

FIG. 4. Dependence of the asymmetry parameter $\beta\left({ }^{3} P \rightarrow{ }^{2} D\right)$ and cross section $\sigma\left({ }^{3} P \rightarrow{ }^{2} D\right)$ for the ${ }^{2} D$ ion term of sulfur on angular momentum transfers $j_{t} \neq l_{0}$ as a function of photoelectron kinetic energy. Left-hand scale refers to (i) the solid line denoting $\beta$ and (ii) the dashed line denoting $\beta\left(j_{t}=l_{0}=1\right)$, both for the ${ }^{3} P \rightarrow{ }^{2} D$ transition. Right-hand scale refers to the dot-dashed line which denotes the ratio $\left[\sigma-\sigma\left(j_{t}=1\right)\right] / \sigma$ for the ${ }^{3} P \rightarrow{ }^{2} D$ transition. 


\section{DISCUSSION AND CONCLUSIONS}

We have shown that anisotropic electron-ion interactions in atomic sulfur lead to measurable differences between photoelectron angular distribution asymmetry parameters corresponding to alternative ionic term levels. Similar effects are expected for most open-shell atoms. A measure of the strength of anisotropic electron-ion interactions is the difference between phase shifts for alternative final-state channels. In atomic sulfur these phase-shift differences are as large as 0.7 rad. A separate study of atomic oxygen ${ }^{7}$ found phase-shift differences of only $0.2 \mathrm{rad}$ and asymmetry parameters that were nearly identical for each ionic term level. However, atomic oxygen and the other second-row elements are regarded as exceptions, since they are too light to have strong interactions, and atomic sulfur is regarded as more typical of open-shell atoms in general. Our choice of atomic sulfur for study was purely a matter of convenience. We know of no experimental data on photoelectron angular distributions for an open-shell atom. We emphasize, however, that we expect the magnitude of the difference between asymmetry parameters and the magnitude of the cross sections to be experimentally measurable for many open-shell atoms.

For the particular case of atomic sulfur we have found that angular momentum transfers $j_{t} \neq l_{0}$, which do not arise in the Cooper-Zare model, ${ }^{4}$ contribute only a small but nevertheless significant amount to the asymmetry parameters and to the cross sections. We simply do not know whether this will hold true for other open-shell atoms. The contributions to the asymmetry parameter from angular momentum transfers $j_{t}=l_{0}$ are, how- ever, quite different from those in the CooperZare model, which has only $j_{t}=l_{0}$ contributions, since the phase-shift differences are so large in the different final-state channels [cf. Eqs. (37b) and $(40 \mathrm{~b})]$.

For closed-shell atoms our formulas reduce rigorously to those of the Cooper-Zare model. Unfortunately, nearly all experimental measurements of photoelectron angular distributions known to us are for closed-shell atoms. Considering the importance of photoelectron angular distributions to such diverse areas as radiation dosimetry (e.g., $\delta$-ray spectrum $)^{30}$ and the physics of the upper atmosphere (e.g., conjugate point phenomena $)^{31}$ as well as to theoretical physics, as emphasized in this paper, we feel that experimental data on photoelectron angular distributions for open-shell atoms would be most valuable.

Last, we point out that our formulas for photoelectron angular distributions have been derived for any electron-ion coupling scheme, but worked out in detail only for $L S$ coupling. In general the electron-ion interaction is best described in an intermediate coupling scheme, particularly in semiempirical calculations. ${ }^{3}$ Nearly all $a b$ initio atomic calculations, however, use the $L S$-coupling scheme, and it is for these calculations that our formulas have been worked out most fully. While we have calculated phase shifts and dipole matrix elements in HF approximation, other more accurate procedures (e.g., many-body perturbation theory, random-phase approximation, etc.) may be used to compute these quantities for use in our formulas for the asymmetry parameter. Similarly, while we have ignored fine-structure splittings of the ionic core, these may easily be included in angular-distribution calculations using our formulas as discussed at the end of Sec. III.
* Supported in part under National Aeronautics and Space Administration Grant No. NGR 28-004-021.

† Supported in part under National Science Foundation Grant No. GP-38905.

${ }^{1}$ U. Fano and D. Dill, Phys. Rev. A $\underline{6}, 185$ (1972).

${ }^{2}$ D. Dill and U. Fano, Phys. Rev. Lett. 29, 1203 (1972).

${ }^{3}$ D. Dill, Phys. Rev. A 7 , 1976 (1973).

${ }^{4} \mathrm{~J}$. Cooper and R. N. Zare, Lectures in Theoretical Physics, edited by S. Geltman, K. T. Mahanthappa, and W. E. Britten (Gordon and Breach, New York, 1969), Vol. XI-C, pp. 317-37.

${ }^{5}$ For representative experimental measurements see, e.g., T. A. Carlson, G. E. McGuire, A. E. Jonas, K. L. Cheng, C. P. Anderson, C. C. Lu, and B. P. Pullen, in Proceedings of an International Conference on Electron Spectroscopy, Asilomar, California, 1971, edited by D. A. Shirley (North-Holland, Amsterdam, 1972), pp. 207-231; P. Mitchell and K. Codling, Phys.
Lett. 38A, 31 (1972); M. J. Lynch, A. B. Gardner, and K. Codling, Phys. Lett. 40A, 349 (1972); A. Niehaus and M. W. Ruf, Z. Physik 252, 84 (1972); M. J. Lynch, K. Codling, and A. B. Gardner, Phys. Lett. 43A, 213 (1973); M. J. Van der Wiel and C. E. Brion, J. Electron Spectrosc. Related Phenomena 1, 439 (1973); G. R. Branton and C. E. Brion, J. Electron Spectrosc. Related Phenomena 3 , 123 (1974).

${ }^{6}$ For representative theoretical calculations see, e.g., J. W. Cooper and S. T. Manson, Phys. Rev. 177, 157 (1969); S. T. Manson and J. W. Cooper, Phys. Rev. A 2 , 2170 (1970); S. T. Manson, Phys. Rev. Lett. 26 219 (1971); D. J. Kennedy and S. T. Manson, Phys. Rev. A 5,227 (1972); S. T. Manson, J. Electron Spectrosc. Related Phenomena 1, 413 (1973); S. T. Manson, Chem. Phys. Lett. 19, 76 (1973).

${ }^{7}$ A. F. Starace, S. T. Manson, and D. J. Kennedy, Phys. Rev. A $\underline{9}, 2453$ (1974). 
${ }^{8} \mathrm{~L}$. Lipsky, in Fifth International Conference on the Physics of Electronic and Atomic Collisions: Abstracts of Papers (Nauka, Leningrad, 1967), pp. 617-18.

${ }^{9}$ L. Lipsky (unpublished).

${ }^{10}$ V. L. Jacobs and P. G. Burke, J. Phys. B $\underline{5}$, L67 (1972).

${ }^{11}$ D. Dill, S. T. Manson, and A. F. Starace, Phys. Rev. Lett. 32, 971 (1974).

${ }^{12}$ C. N. Yang, Phys. Rev. 74, 764 (1948).

${ }^{13}$ See Ref. 6, first paper.

${ }^{14}$ J. A. R. Samson, J. Opt. Soc. Am. 59, 356 (1969); Philos. Trans. R. Soc. Lond. A 268, 141 (1970); J. A. R. Samson and J. L. Gardner, J. Opt. Soc. Am. $\underline{62}, 856$ (1972).

${ }^{15}$ V. Schmidt, Phys. Lett. 45A, 63 (1973).

${ }^{16} \mathrm{U}$. Fano and G. Racah, Irreducible Tensorial Sets (Academic, New York, 1959), Chap. 12.

${ }^{17}$ A. P. Yutsis, I. B. Levinson, and V. V. Vanagas, Theory of Angular Momentum (Israel Program for Scientific Translations, Jerusalem, 1962), Chapter V.

${ }^{18}$ J. S. Briggs, Rev. Mod. Phys. $\underline{43}, 189$ (1971).
${ }^{19}$ Reference 16, Eq. (15.7).

${ }^{20}$ Reference 16, Eq. (15.7').

${ }^{21}$ U. Fano, Phys. Rev. 140, A67 (1965).

${ }^{22}$ B. W. Shore and D. H. Menzel, Principles of Atomic Spectra (Wiley, New York, 1968), Eq. (10.4).

${ }^{23}$ See Ref. (16), Eq. (I.2) or Ref. (17), Eq. (A6.53).

${ }^{24}$ M. J. Conneely, K. Smith, and L. Lipsky, J. Phys. B 3, 493 (1970).

${ }^{25}$ A.Dalgarno, R. J. W. Henry, and A. L. Stewart, Planet. Space Sci. 12, 235 (1964).

${ }^{26}$ E. Clementi and C. Roetti, At. Data Nucl. Data Tables 14,177 (1974).

${ }^{27} \bar{F}$. Herman and S. Skillman, Atomic Structure Calculations (Prentice-Hall, Englewood Cliffs, N. J., 1963).

${ }^{28}$ A. F. Starace, Phys. Rev. A $\underline{3}, 1242$ (1971); $\underline{8}, 1141$ (1973).

${ }^{29}$ J. W. Cooper, Phys. Rev. 128, 681 (1962).

${ }^{30} \mathrm{H}$. Bichsel (private communication).

${ }^{31}$ S. T. Manson, D. J. Kennedy, A. F. Starace, and D. Dill, Planet. Space Sci. 22, 1535 (1974). 